\title{
Design of an Analog Front End for a Bio-Inspired Auditory Sensor of a Novel Totally Implantable Cochlear Implant
}

\author{
Jeong Hoan Park, Jin Ho Kim, Yoon-Kyu Song1,*, Youngdo Jung², \\ Shin $\mathrm{Hur}^{2}$, Wandoo $\mathrm{Kim}^{2}$ and Sung June Kim${ }^{* *}$ \\ Department of Electrical and Computer Science, Seoul National University, \\ 1 Gwanak, Gwanak-ro, Gwanak-gu, Seoul 151-744, Korea \\ ${ }^{1}$ Graduate School of Convergence Science and Technology, Seoul National University, \\ 864-1 Iui-dong, Yeongtong-gu, Suwon-si, Gyeonggi-do 443-270, Korea \\ ${ }^{2}$ Korea Institute of Machinery \& Materials, \\ 156 Gajeongbuk-Ro, Yuseong-Gu, Daejeon 305-343, Korea
}

(Received December 17, 2012; accepted April 12, 2013)

Key words: cochlear implant, totally implantable cochlear implant, artificial basilar membrane, piezoelectric sensor, low-noise low-power analog front end, biosensor

In this paper, we present a low-power and low-noise analog front end for a totally implantable cochlear implant using a bio-inspired auditory sensor. We used the " $g_{\mathrm{m}}$ of $I_{\mathrm{D}}$ " method to design an analog front end as the interface to an artificial basilar membrane with reduced flicker and thermal noises and low power consumption. We fabricated an Application-Specific Integrated Circuit (ASIC) chip with an analog front end using a $0.35 \mu \mathrm{m}$ high-voltage CMOS process, showing a measured gain range of 40.35-62.94 $\mathrm{dB}$ with an input-referred noise of $5.32 \mu \mathrm{Vrms}$ at a power consumption of $272 \mu \mathrm{W}$ per channel. As proof of concept demonstration, we used an analog front end with an artificial basilar membrane sensor, exhibiting an audio signal transduction suitable for a biomimetic artificial cochlear implant.

\section{Introduction}

The cochlear implant, which is used to restore the hearing ability of patients with sensorineural hearing loss, is one of the most successful neural prostheses; the system consists of two units, an external unit and an internal unit. ${ }^{(1-4)}$ The external unit is responsible for sound pickup and speech processing, with the internal unit receiving the telemetry signal sent by the external unit and producing analog waveforms suitable for

*Corresponding author: e-mail: songyk@snu.ac.kr

${ }^{* *}$ Corresponding author: e-mail: kimsj@snu.ac.kr 
exciting the remaining auditory neurons in such patients. Recipients of conventional cochlear implants cannot use the device in some environments, such as when taking a shower, playing water sports, or sleeping owing to the existence of the external unit. Furthermore, patients cannot conceal their deafness owing to the external device. For these reasons, patients need a totally implantable cochlear implant device that requires no external hardware during its use. All of the functions should be handled by the internal unit, thus making the system extremely convenient to wear, not causing a cosmetic problem. Thus, a totally implantable cochlear implant is the future goal of cochlear implant developers in terms of both recipient convenience and technological innovation. In 2008, Cochlear Ltd. (Australia) developed a prototype of a totally implantable cochlear implant, which has a package-mounted electric microphone, sound processing electronics, and a rechargeable battery. ${ }^{(5)}$ It showed some limitations, however.

Firstly, speech perception scores were significantly lower than those of conventional units owing to the reduced sensitivity of the subcutaneous microphone and body noise interference such as the blood stream and flying hair. Secondly, the estimated battery lifetime of the developed system was about six years owing to the relatively high power consumption of the sound processing unit. Moreover, the system developed was bulky and heavier than conventional systems.

To solve these problems, we proposed a totally implantable advanced cochlear implant using a bio-inspired auditory sensor or an artificial basilar membrane, as shown in Fig. 1. A bio-inspired auditory sensor or an artificial basilar membrane that mimics the biological cochlea is the most promising solution for an advanced totally implantable cochlear implant..$^{(6-10)}$ It replaces the external microphone and speech processor with one tiny mechanical sensor, while maintaining a low-power consumption and a compact size. The bio-inspired auditory sensor based on the piezoelectric principle produces electrical signals of multiple channels in response to the spectrum of acoustic inputs. In the literature, we found that many people used sensors that stimulate auditory nerves directly. ${ }^{(6,7)}$ However, such sensors cannot produce charge-balanced electrical signals to stimulate auditory nerves in response to the same acoustic inputs regardless of their position, material, and geometric structure, which might lead to safety issues. ${ }^{(3)}$ Hence, we proposed the use of output signals of the sensor, not as auditory nerve stimulators but as an ultra low-noise and ultra low-power digital signal processor. An additional electrical module with a digital controller and a biphasic current stimulator uses output signals of the sensor and delivers charge-balanced biphasic current pulses to auditory nerves. The digitization of the output signals using analog-to-digital converter (ADC) is required for the digital controller to recognize the signals from the sensor. However, the output signal range of the sensor is $0.1-10 \mathrm{mV},{ }^{(6-10)}$ which is too small to be easily digitized; thus, a suitable analog front end or preamplifier array should be included in the system. The analog front end should have a low-noise design topology to guarantee a reasonable signal-to-noise ratio from the minute sensor signal. Also, low-power consumption should be considered so as not to increase the surrounding temperature too much, in order to prevent harmful thermal effects such as tissue damage in the surrounding area.

In this work, we implemented a low-noise, low-power analog front end prototype interfacing with a bio-inspired auditory sensor as an initial study of the design 


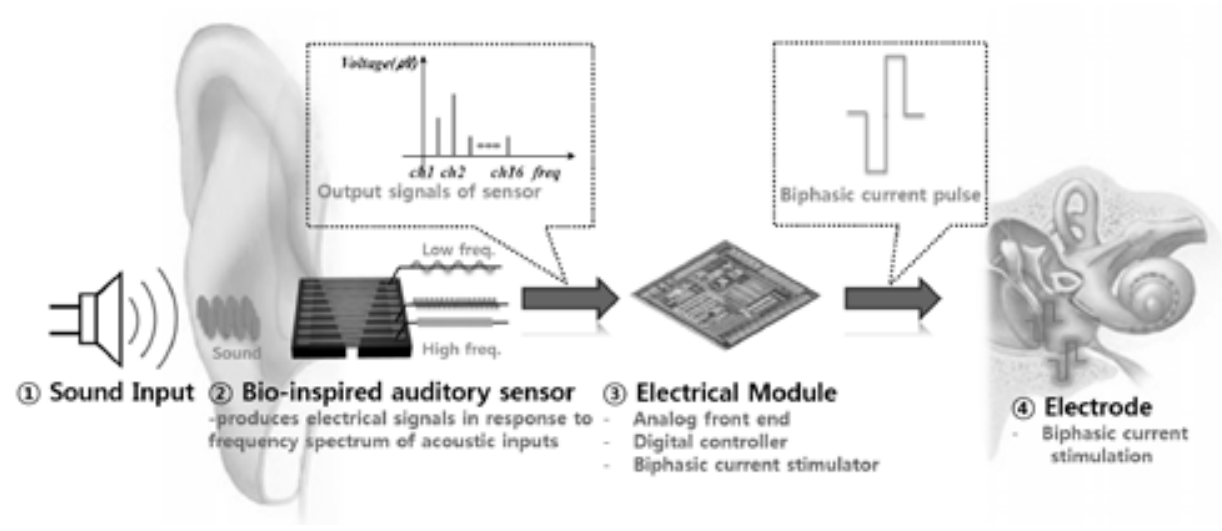

Fig. 1. Schematic overview of totally implantable advanced cochlear implant using bio-inspired auditory sensor.

of electrical modules for a bio-inspired auditory sensor. Before designing the analog front end, we considered specifications of the analog front end, e.g., gain, bandwidth, power consumption, and input-referred noise based on the output signal of the sensor, which resulted in a totally implantable cochlear implant having suitable signal qualities and a long battery life. Then, we developed an optimal design procedure and applied it to the fabrication of an AISC in a high-voltage CMOS process. A bench-top test and test interfacing with the sensor were also implemented; their results were close to the simulation results.

For detailed information on the analog front end, this paper is organized as follows. The concept of the bio-inspired auditory sensor and the design consideration of the analog front end are described in $\S 2$. The circuit description, design procedure, and characterization of the designed chip are detailed in $\S 3$. Test results and conclusion are presented in $\S \S 4$ and 5, respectively.

\section{Design Consideration}

\subsection{Bio-inspired auditory sensor}

The bio-inspired auditory sensor is composed of a silicon substrate and a piezoelectric material such as a polyvinylidene fluoride (PVDF) film between channel metal patterns and the reference metal pattern, as shown in Fig. 2(a). ${ }^{(8)}$

Each silicon substrate region under the channel is etched with different lengths; therefore, all channels have their own resonant frequency. When a compound acoustic signal vibrates the PVDF film, as shown in Fig. 2(b), the PVDF film generates voltage differences between channel patterns and the reference pattern, and each electrical signal responds to its resonant frequency; that is, the long-channel metal pattern responds to a 


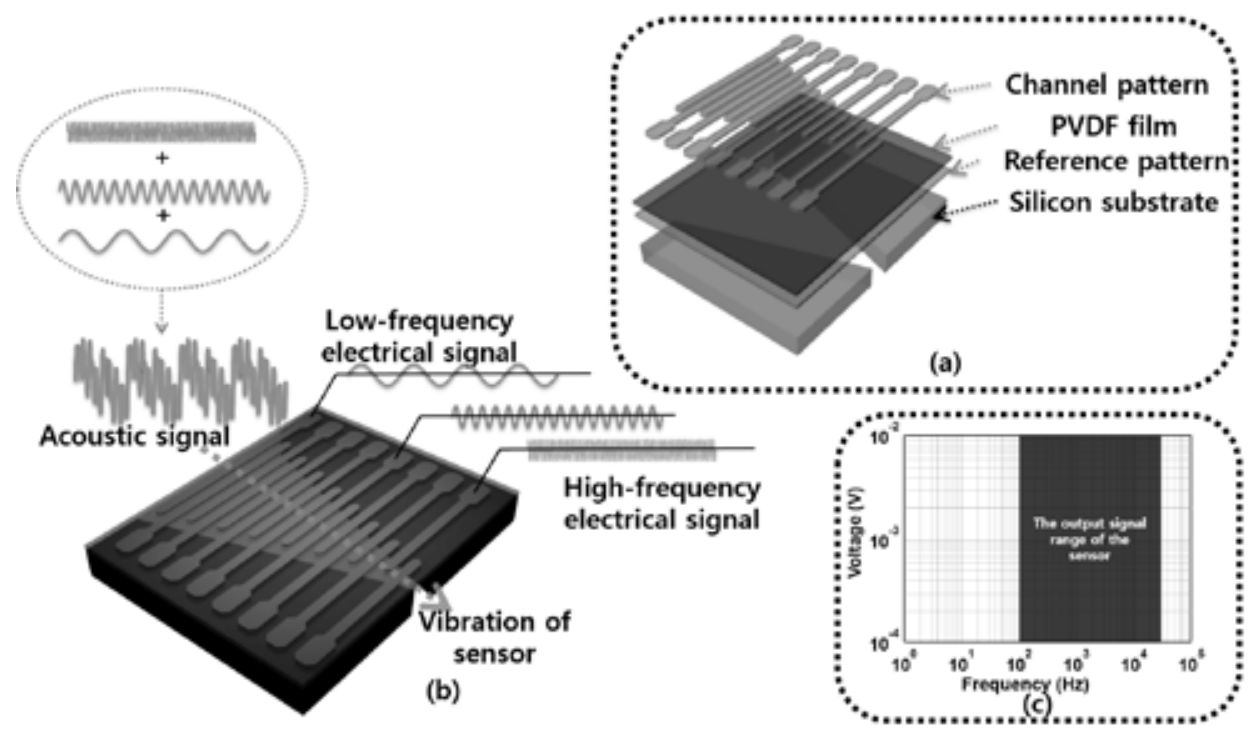

Fig. 2. PVDF-based bio-inspired auditory sensor: (a) components, (b) operational concept, and (c) output signal range of the sensor.

lower frequency of the acoustic signal, while the short-channel metal pattern responds to a higher frequency. Thus, the sensor has the frequency decomposition properties of a biological basilar membrane.

Figure 2(c) shows the output range of sensors, which is the primary consideration of analog front end specifications, reported elsewhere. ${ }^{(7-9)}$ Inaoka et al. developed an artificial cochlear epithelium using PVDF, which produces an output voltage of $5 \mathrm{mV}$ with a bandwidth range of $1-40 \mathrm{kHz}$ in response to $100 \mathrm{~dB}$ sound pressure level (SPL). ${ }^{(7)}$ Hur et al. also developed a macroscale biomimetic cantilever acoustic sensor based on a lead magnesium niobate-lead titanate $\left[\mathrm{Pb}\left(\mathrm{Mg}_{1 / 3} \mathrm{Nb}_{2 / 3}\right) \mathrm{O}_{3}-\mathrm{PbTiO}_{3}, \mathrm{PMN}-\mathrm{PT}\right]$ piezoelectric material that generates an output voltage range of $0.1-100 \mathrm{mV}$ with a bandwidth range of $490 \mathrm{~Hz}-13.6 \mathrm{kHz}$ in response to $94 \mathrm{~dB}$ SPL. ${ }^{(9)}$

\subsection{Analog front end for the bio-inspired auditory sensor}

On the basis of the electrical output ranges of the sensors mentioned above, we considered analog front end specifications that are gain, bandwidth, power consumption, and input-referred noise in detail. We estimate that the analog front end should have a gain of at least $40 \mathrm{~dB}$, which leads to the maximum output signal of the sensor to be about half of the supply voltage, thus reducing errors of converting analog signals to digital signals in the ADC. A bandwidth of $20 \mathrm{kHz}$ is determined to handle the audible frequency range. 
Power consumption is also a very important specification of the analog front end. Assuming that an eight-channel amplifier array, ${ }^{(11)}$ which is the minimum number of stimulation channels for a reasonable speech perception score, is used for $8 \mathrm{~h}$ per day for 10 years with a $100 \mathrm{mAh}$ rechargeable battery, the maximum allowable power consumption per channel amplifier is $428 \mu \mathrm{W}$. Moreover, on the basis of the output range of the sensor, the input-referred noise should not exceed $10 \mu \mathrm{Vrms}$ to maintain the SNR greater than $20 \mathrm{~dB}$. Compared with a fixed gain amplifier, a variable gain amplifier (VGA) can accommodate multiple types of sensor since the electrical output of the sensor depends on the type of piezoelectric material and its geometric structure. Also, a VGA can compensate for the fluctuation in the sensitivity of the sensor due to the variation in the surgical implementation of the sensors. The target parameters of the analog front end for the sensor are summarized in Table 1.

\section{Circuit Description}

\subsection{Overall system}

Figure 3(a) shows the overall architecture of the designed analog front end for the bio-inspired auditory sensor. The analog front end contains multichannel preamplifiers, an analog multiplexer (AMUX), and a VGA. Preamplifiers amplify the electrical outputs of the sensor with a constant gain. The amplified outputs of preamplifiers are multiplexed to the VGA, which provides gains that range from 1 to $15 \mathrm{~V} / \mathrm{V}$ in 15 linear steps. Thus, the overall gain of the analog front end can be controlled from 40 to 63.5 $\mathrm{dB}$ according to user-provided digital input.

Table 1

Target parameters of analog front end.

\begin{tabular}{lc}
\hline Preamplifier & \\
\hline Parameter & Target value \\
\hline Gain & $40 \mathrm{~dB}$ \\
Bandwidth & $20 \mathrm{~Hz}-20 \mathrm{kHz}$ \\
Input-referred noise & $<10 \mu \mathrm{Vrms}$ \\
Common mode rejection ratio (CMRR) & $>60 \mathrm{~dB}$ \\
Power supply rejection ratio (PSRR) & $>60 \mathrm{~dB}$ \\
Power consumption & $<500 \mu \mathrm{W}$ \\
Area & $<0.15 \mathrm{~mm}^{2}$ \\
\hline VGA & \\
\hline Parameter & Target value \\
\hline Gain & $1-15 \mathrm{~V} / \mathrm{V}$ \\
Power consumption & $<500 \mu \mathrm{W}$ \\
Area & $<0.15 \mathrm{~mm}^{2}$ \\
\hline
\end{tabular}



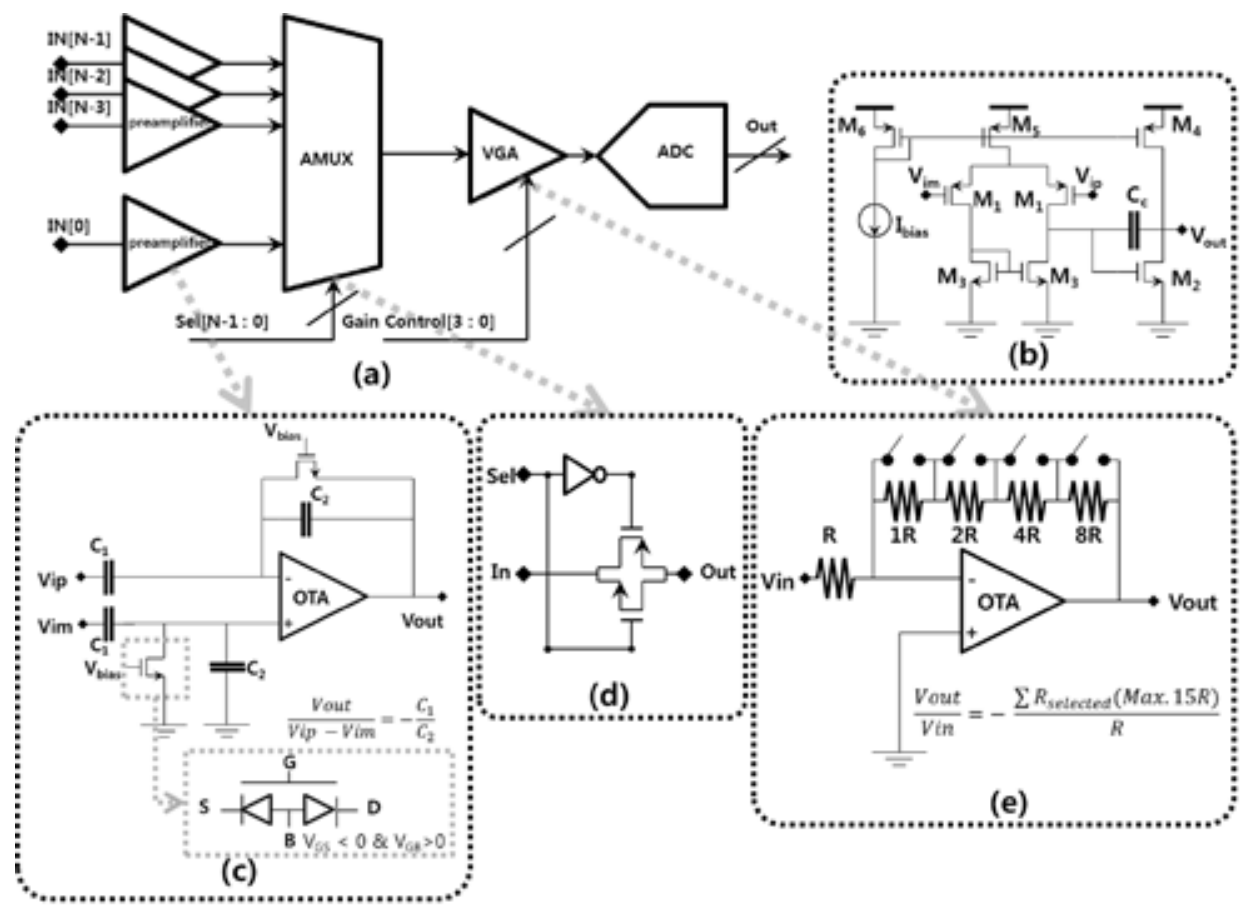

Fig. 3. Block diagram of design of analog front end: (a) system diagram, (b) schematic of OTA, (c) schematic of preamplifier, (d) AMUX, and (e) schematic of VGA.

\subsection{Operational transconductance amplifier (OTA)}

The core OTA design for the preamplifier and the VGA is very important to reduce both the power consumption and the thermal and flicker noises. We adopted the Miller OTA structure, which provides a large output swing and a large open-loop gain to be suitable for feedback, as shown in Fig. 3(b). The input-referred thermal and flicker noise power spectrum densities of the Miller OTA are respectively expressed as

$$
\begin{gathered}
\frac{\bar{V}_{\mathrm{ni}, \text { thermal }}^{2}}{\Delta f}=\frac{8 \mathrm{kT} \gamma}{g_{\mathrm{M} 1}^{2}}\left(g_{\mathrm{M} 1}+g_{\mathrm{M} 3}\right), \\
\frac{\bar{V}_{\mathrm{ni}, \text { flicker }}^{2}}{\Delta f}=\frac{2 \kappa_{\mathrm{p}}}{C_{\mathrm{ox}}(W L)_{1} f}+\frac{2 \kappa_{\mathrm{n}}}{C_{\mathrm{ox}}(W L)_{3} f}\left(\frac{g_{\mathrm{M} 3}}{g_{\mathrm{M} 1}}\right)^{2},
\end{gathered}
$$

where $\mathrm{k}$ is the Boltzmann constant, $\mathrm{T}$ is the absolute temperature, $\gamma$ is $2 / 3$ for longchannel devices, $g_{\mathrm{M} i}$ is the transconductance of $\mathrm{M} i$ transistor, $(W L)_{i}$ is the product of the 
width of the $\mathrm{M} i$ transistor $\left(W_{i}\right)$ and the length of the $\mathrm{M} i$ transistor $\left(L_{i}\right)$, and $\kappa_{\mathrm{p}}$ and $\kappa_{\mathrm{n}}$ are process-dependent constants. From eqs. (1) and (2), the input-referred noise power is determined according to the relationship between $g_{\mathrm{M} 1}, g_{\mathrm{M} 3}$ and $(W L)_{1},(W L)_{3}$, in which larger values of $g_{\mathrm{M} 1}, W_{1}, W_{3}, L_{1}$ and $L_{3}$ and a smaller value of $g_{\mathrm{M} 3}$ result in a lower inputreferred noise. The detailed design procedure for the Miller OTA is described in Fig. 4 based on eqs. (1) and (2) to optimize the tradeoff between the power consumption and the input-referred noise power spectrum density. ${ }^{(12)}$

First, we set the maximum input-referred noise of the OTA and the minimum $g_{\mathrm{M} 1}$ from eq. (1). Provided that $\mathrm{M} 1$ is biased in the deep weak-inversion region $\left(g_{\mathrm{M} 1} / I_{\mathrm{D} 1}>20\right)$, we can obtain a higher transconductance efficiency to achieve a low-power consumption design, while reducing flicker noise significantly owing to the high gate capacitance of M1 $\left[(W L)_{1}\right]$. Second, we select an appropriate $C_{\mathrm{c}}$ in order to determine the bandwidth, and we set $g_{\mathrm{M} 2}$ at a value tenfold that of $g_{\mathrm{M} 1}$ to ensure stability. M2 is biased in the weakinversion region for the same reason as M1. Last, we choose $g_{\mathrm{M} 3}$ such that $g_{\mathrm{M} 3} / I_{\mathrm{D} 3}=g_{\mathrm{M} 2} / I_{\mathrm{D} 2}$ to prevent mismatches and set the remaining parameters. The input-referred noise can be higher than our target value because we ignore the flicker noise in eq. (2). Thus, once we set a $g_{\mathrm{M} 1}$ smaller than the previous $g_{\mathrm{M} 1}$ and repeat this procedure, we will have an optimized Miller OTA. The designed dimensions and operating points of each transistor are listed in Table 2.

\subsection{Preamplifier}

The preamplifier is operated using an AC-coupled high-pass filter with MOS deep depletion region pseudo-resistors as feedback elements, as shown in Fig. 2(c). ${ }^{(13,14)}$ This structure allows the preamplifier to not only block the DC offset and voltage drift of the

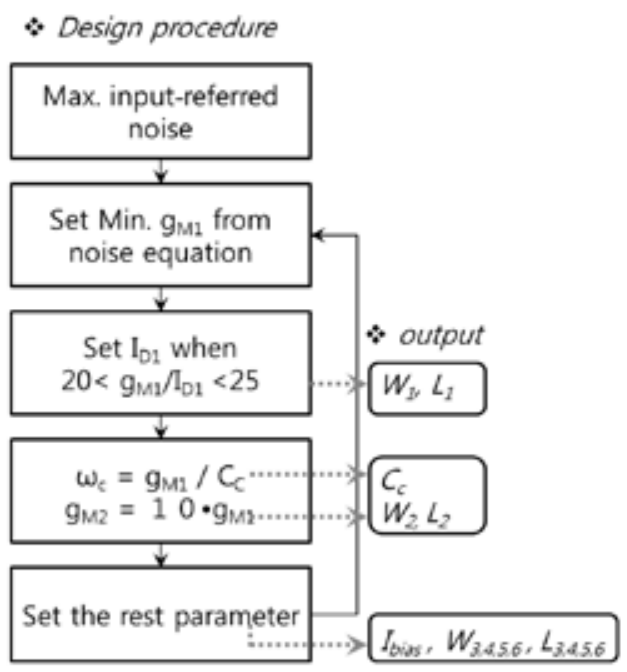

Fig. 4. Design procedure for Miller OTA. 
Table 2

Operating parameters of Miller OTA transistors.

\begin{tabular}{lcccc}
\hline & $g_{\mathrm{M}}(\mu \mathrm{A} / \mathrm{V})$ & $I_{\mathrm{D}}(\mu \mathrm{A})$ & $g_{\mathrm{M}} / I_{\mathrm{D}}\left(\mathrm{V}^{-1}\right)$ & $W / L(\mu \mathrm{m} / \mu \mathrm{m})$ \\
\hline M1 & 115 & 6.3 & 18.2 & $95.4 / 1$ \\
M2 & 758 & 34.1 & 22.24 & $912 / 0.5$ \\
M3 & 131 & 6.3 & 20.83 & $203.2 / 1$ \\
M4 & 195 & 34.1 & 5.7 & $10 / 0.5$ \\
M5 & 74.7 & 12.8 & 5.84 & $8.4 / 1$ \\
M6 & 54.8 & 9.17 & 5.98 & $6 / 1$ \\
\hline
\end{tabular}

electrode but also integrate passive elements into the ASIC. The mid-band gain of the preamplifier is given by $-C_{1} / C_{2}$. The large resistance of a pseudo-resistor composed of MOS in the deep depletion region makes the high-pass corner frequency fall below 10 $\mathrm{Hz}$, and the bandwidth of the OTA determines the low-pass corner frequency to be 20 $\mathrm{kHz}$.

\subsection{Analog multiplexer (AMUX)}

The amplified outputs of preamplifiers are multiplexed by the AMUX on the basis of digital inputs (Sel [N-1:0]). The structure of the AMUX shown in Fig. 3(d) is a complementary pair shunt-switch for minimizing signal distortion.

\subsection{Variable gain amplifier (VGA)}

The multiplexed signal is further amplified by the VGA to respond to multiple types of sensor. As shown in Fig. 3(e), the structure of the VGA is an inverting amplifier whose feedback resistor can be controlled linearly by four-bit digital inputs (Gain Control [3:0]). The VGA has a gain range of 1 to $15 \mathrm{~V} / \mathrm{V}$.

\section{Measurement Results}

\subsection{Simulation and measurement results}

To validate the designed analog front end, we simulate and fabricate a prototype chip consisting of two preamplifiers, one AMUX and one VGA, in the Dongbu $0.35 \mu \mathrm{m}$ highvoltage CMOS process (Bipolar-CMOS-DMOS or BCDMOS). It occupies a total area of $0.34 \mathrm{~mm}^{2}$, as shown in Fig. 5. The simulation is performed using BSIM3V3 transistor models, and the measurement is performed using a signal analyzer (HP35670A, Agilent Technologies, Inc., USA) and an oscilloscope (DPO 4034, Tektronix, Inc., USA).

Concerning the preamplifier, the simulation and measurement results are 39.85 and $39.37 \mathrm{~dB}$ for the mid-band gains and 27.5 and $37 \mathrm{kHz}$ for the bandwidth, respectively, as shown in Fig. 6(a) (when $C_{1}=12.7 \mathrm{pF}$ and $C_{2}=0.13 \mathrm{pF}$ ).

Although the high-pass corner frequency shows a mismatch between the simulation and measurement data due to the use of inaccurate pseudo-resistors, this does not affect the circuit performance in terms of the rejection of the DC offset and the voltage drift from the electrode. 


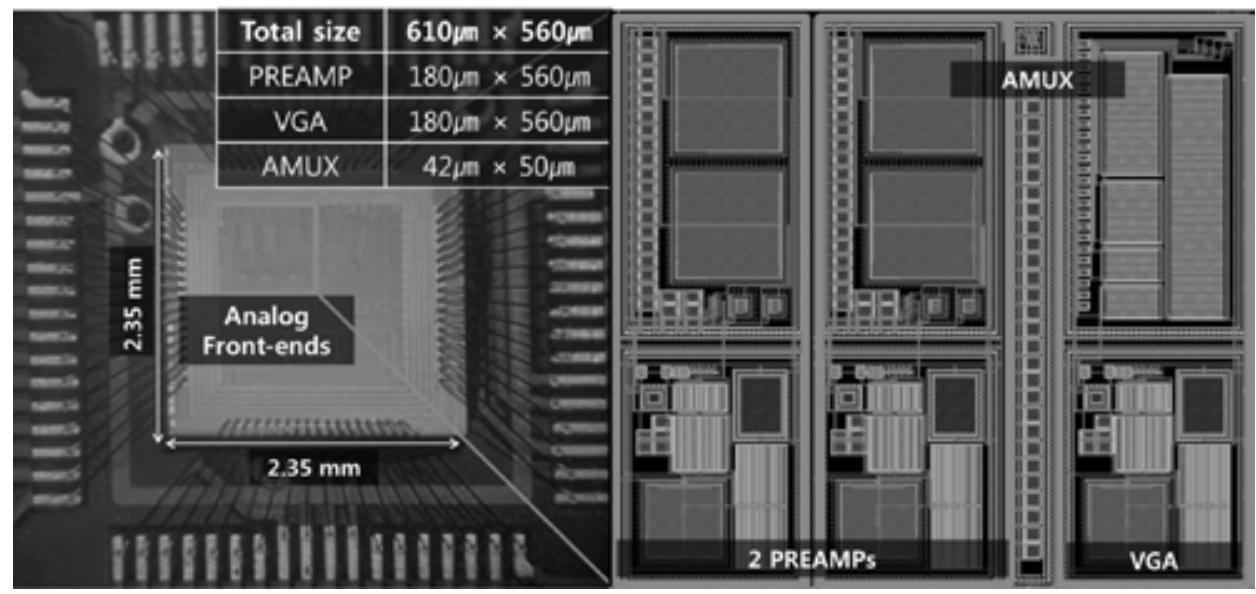

Fig. 5. Layout of fabricated ASIC chip of analog front end.

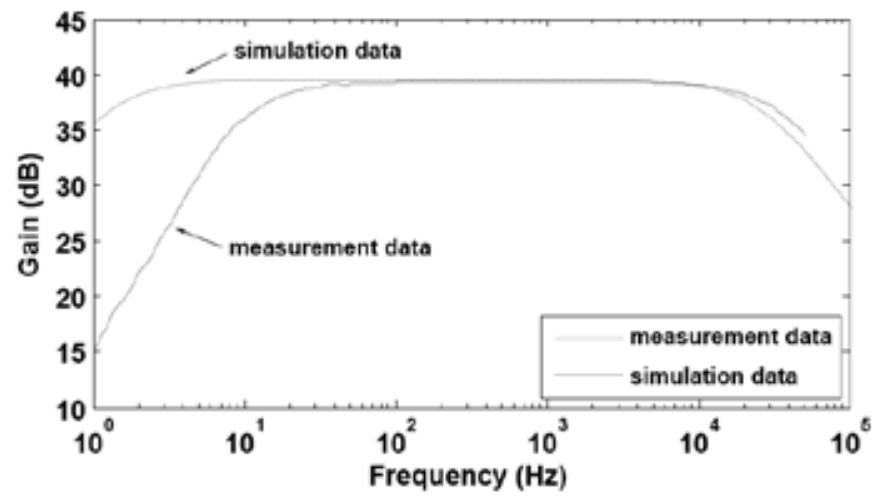

(a)

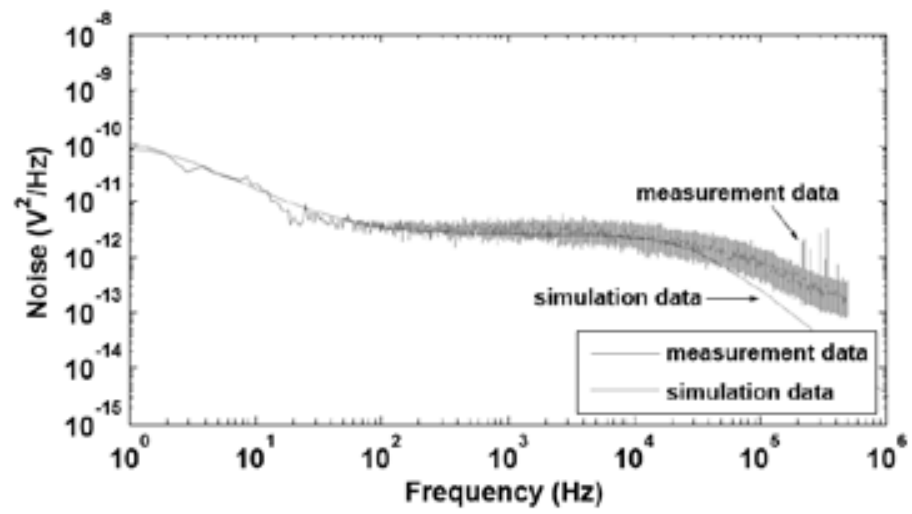

(b)

Fig. 6. Benchtop test results of preamplifier (a) gain [39.85 dB (simulation), $39.37 \mathrm{~dB}$ (measurement)] and bandwidth [27.5 kHz (simulation), $37 \mathrm{kHz}$ (measurement)]. (b) Output noise power spectrum density [input-referred noise: $3.66 \mu \mathrm{Vrms}$ (simulation), $5.32 \mu \mathrm{Vrms}$ (measurement)]. 
The input-referred noise values, obtained by dividing the sum of an output noise power spectrum density range of $1 \mathrm{~Hz}-50 \mathrm{kHz}$ by the squared amplifier mid-band gain, are $3.66 \mu \mathrm{Vrms}$ from the simulation data and $5.32 \mu \mathrm{Vrms}$ from the measurement data, as shown in Fig. 6(b).

Figure 7 shows the simulation and measurement gain responses with a linear change in response to the digital inputs (Gain Control [3:0]).

Table 3 shows a brief summary of the simulation and measurement results of the analog front end, which shows a close match between the target values in the simulation and measurement values.

\subsection{Test interfacing with bio-inspired auditory sensor}

The fabricated chip was tested by interfacing with a macro scale bio-inspired auditory sensor prototype fabricated at the Korea Institute of Machinery and Materials, which is a modified version that was tested for frequency decomposition by Inaoka et al. ${ }^{(8)}$ To show the gain of the analog front end interfacing with the sensor, we measured output signals of the sensor and output of the analog front end, as shown in Fig. 8(a). The speaker or 'mouth simulator' (Type 4227, Brüel \& Kjær Sound \& Vibration Measurement A/S, Denmark) stimulates a macroscale bio-inspired auditory sensor with a sinusoidal sound pressure of $94 \mathrm{~dB}$ SPL and a frequency of $1 \mathrm{kHz}$. As a result, the sensor generates electrical signals, and we measure the electrical signal of one channel at a resonant frequency of $1 \mathrm{kHz}$ using a metal probe tip connected to the channel pad of the sensor. In the future, we are considering connecting the channel pad and the input of the analog front end with wire bonding or flip-chip bonding in a monolithic encapsulation package using a liquid crystal polymer (LCP), which will enable the fabrication of a flexible PCB board integrated with all electrical parts and showing a good characteristic of hermetic sealing for the future totally implantable cochlear implant. ${ }^{(15)}$ The electrical signal is amplified by setting the gain to $40 \mathrm{~dB}$. Both the input and output signals are measured by an oscilloscope (DPO 4034, Tektronix, Inc., USA). Furthermore, a commercial differential amplifier (ADA400A, Tektronix, Inc., USA) is used to obtain an accurate input signal.

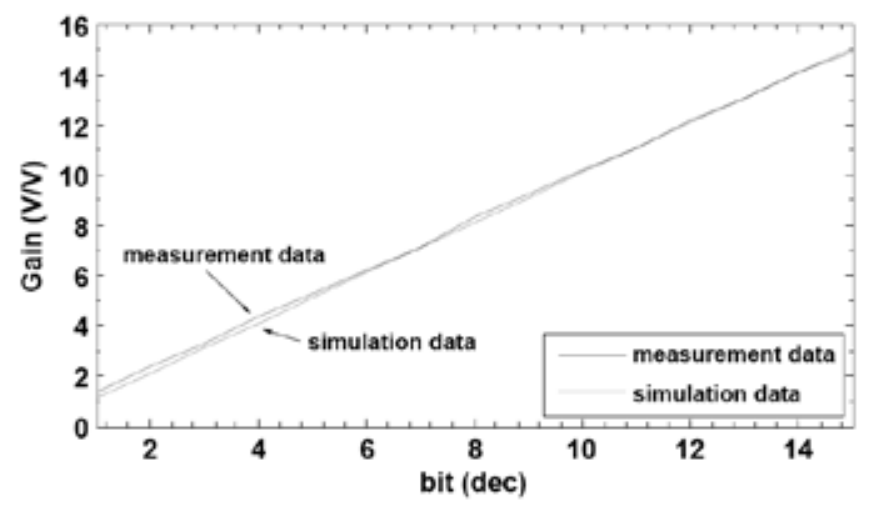

Fig. 7. Gain characteristic of VGA. 
Table 3

Performance characteristics of analog front end.

\begin{tabular}{lccc}
\hline Preamplifier & \multicolumn{3}{c}{} \\
\hline Parameter & Target value & Simulation value & Measurement value \\
\hline Gain & $40 \mathrm{~dB}$ & $39.85 \mathrm{~dB}$ & $39.37 \mathrm{~dB}$ \\
Bandwidth & $20 \mathrm{~Hz}-20 \mathrm{kHz}$ & $5 \mathrm{~Hz}-27.5 \mathrm{kHz}$ & $10 \mathrm{~Hz}-37 \mathrm{kHz}$ \\
Input-referred noise & $<10 \mu \mathrm{Vrms}$ & $3.66 \mu \mathrm{Vrms}(1-50 \mathrm{kHz})$ & $5.32 \mu \mathrm{Vrms}(1-50 \mathrm{kHz})$ \\
CMRR & $>60 \mathrm{~dB}$ & $102.5 \mathrm{~dB}$ & $59.82 \mathrm{~dB}$ \\
PSRR & $>60 \mathrm{~dB}$ & $84.2 \mathrm{~dB}$ & $65.8 \mathrm{~dB}$ \\
Power consumption & $<500 \mu \mathrm{W}$ & $221 \mu \mathrm{W}$ & $242 \mu \mathrm{W}$ \\
Area & $<0.15 \mathrm{~mm}^{2}$ & $0.1 \mathrm{~mm}^{2}$ & $0.1 \mathrm{~mm}^{2}$ \\
\hline VGA & & & \\
\hline Parameter & Target value & Simulation value & Measurement value \\
\hline Gain & $1-15 \mathrm{~V} / \mathrm{V}$ & $1.37-15.0 \mathrm{~V} / \mathrm{V}$ & $1.12-15.1 \mathrm{~V} / \mathrm{V}$ \\
Power consumption & $<500 \mu \mathrm{W}$ & $221 \mu \mathrm{W}$ & $242 \mu \mathrm{W}$ \\
Area & $<0.15 \mathrm{~mm}^{2}$ & $0.1 \mathrm{~mm}^{2}$ & $0.1 \mathrm{~mm}^{2}$ \\
\hline
\end{tabular}

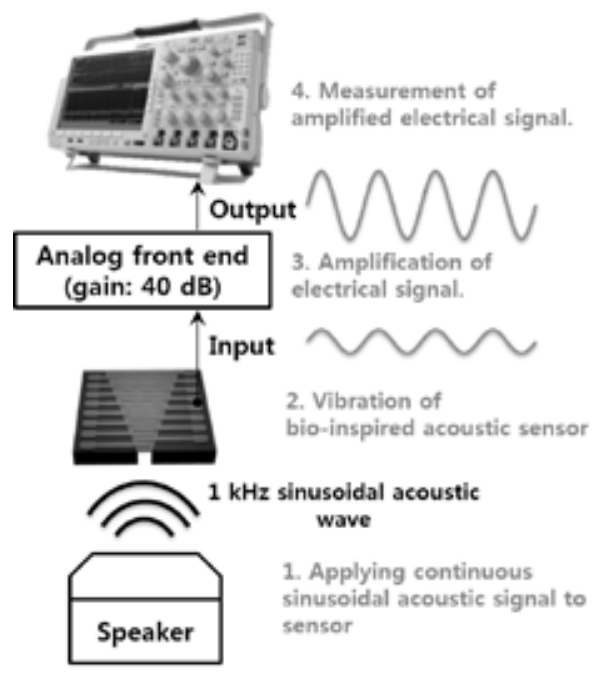

(a)

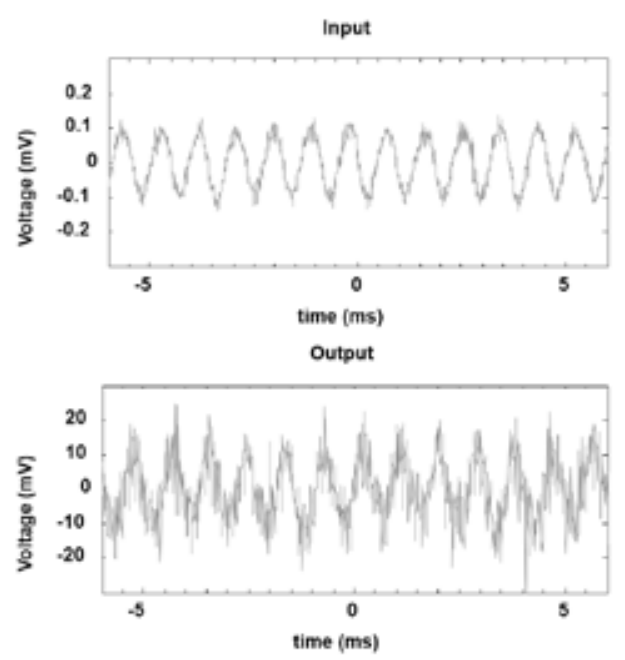

(b)

Fig. 8. Test result of analog front end combined with bio-inspired auditory sensor: (a) experimental setup and (b) test result (The input of the analog front end is measured using a commercial amplifier. The corner frequency is $100 \mathrm{~Hz}$ ). 
Because some power-line noise of the speaker affected the outputs of the sensor due to electromagnetic interference, we used high-pass filtering software in the output waveform, which has a corner frequency of $100 \mathrm{~Hz}$, to reject the noise by postprocessing. Figure $8(\mathrm{~b})$ shows the sound input and measured output signal with a gain of $43 \mathrm{~dB}$ and a frequency of $1 \mathrm{kHz}$. To observe the difference in the gain between other channels, we tested another channel with a resonant frequency of $780 \mathrm{~Hz}$, while stimulating with a sinusoidal sound with a pressure of $94 \mathrm{~dB}$ and a frequency of $780 \mathrm{~Hz}$. We assume that the signals from two channels are enough to show a similar gain of the analog front end, because all equivalent circuits of the sensor are presumably identical and there are hardly any differences in the design parameters among all the channels. The measurement gain of the analog front end is $40.18 \mathrm{~dB}$; thus, all measurement gains of the channel output are almost the same. Unfortunately, we could only see the gain of the analog front end, because of the limited number of preamplifiers and the inadequateness of the digital controller in our prototype ASIC; thus, we did not observe frequency decomposition in the time domain. It would be our next goal to modify the digital controller, integrate more channels in the analog front end chip, and optimize parameters for the bio-inspired auditory sensor, which would enable real-time frequency decomposition with a minimal interchannel crosstalk.

\section{Conclusions}

In this study, we have demonstrated a low-noise analog front end interfaced with a bio-inspired auditory sensor of a novel totally implantable cochlear implant. With the low-noise design of the analog front end using the " $g_{\mathrm{m}}$ of $I_{\mathrm{D}}$ " method, we fabricated a prototype chip that showed a low input-referred noise of $5.32 \mu \mathrm{Vrms}$ and an optimal power consumption of $272 \mu \mathrm{W}$ per channel featuring a good noise-power trade-off. Furthermore, we designed a circuit with a fully tunable variable gain, which made the analog front end respond to multiple types of sensor using different piezoelectric materials and geometries.

When interfaced with the bio-inspired auditory sensory prototype that we fabricated, our circuit performed as expected. In the future, we plan to develop a totally implantable cochlear implant system with a bio-inspired auditory sensor, enhanced in terms of size and performance using the current design of the analog front end.

\section{Acknowledgements}

This work was supported by the Pioneer Project (20120001038) of the Ministry of Education, Science and Technology of Korea. We acknowledge the support of the IC Design Education Center (IDEC) at KAIST, the Technology Innovation Program (10033657) of the Ministry of Knowledge Economy (MKE), and the Inter-university Semiconductor Research Center (ISRC) at Seoul National University. This study was also supported in part by the Brain Korea 21 Project and the Department of Electrical Engineering at Seoul National University in 2012. 


\section{References}

1 B. S. Wilson, C. C. Finley, D. T. Lawson, R. D. Wolford, D. K. Eddington and W. M. Rabinowitz: Nature 352 (1991) 236.

2 B. S. Wilson and M. F. Dorman: J. Rehabil. Res. Dev. 45 (2008) 695.

3 F. G. Zeng, S. Rebscher, W. Harrison, X. Sun and H. Feng: IEEE Rev. Biomed. Eng. 1 (2008) 115.

4 G. Fayad and B. Elmiyeh: Artificial Organs, ed. N. S. Hakim (Springer, London, 2009) Chap. 8.

5 R. J. S. Briggs, H. C. Eder, P. M. Seligman, R. S. C. Cowan, K. L. Plant, J. Dalton, D. K. Money and J. F. Patrick: Otol. Neurotol. 29 (2008) 114.

6 N. Mukherjee, R. D. Roseman and J. P. Willging: J. Biomed. Mater. Res. 53 (2000) 181.

7 S. Hur, H. S. Choi, K. H. Lee and W. D. Kim: Elastom. Composit. 44 (2009) 98.

8 T. Inaoka, H. Shintaku, T. Nakagawa, S. Kawano, H. Ogita, T. Sakamoto, S. Hamanishi, H. Wada and J. Ito: Proc. Natl. Acad. Sci. U.S.A. 108 (2011) 18390.

9 S. Hur, J.-H. Kwak, Y. Jung and Y. H. Lee: IEICE Electron. Exp. 9 (2012) 945.

10 P. Beccue, J. Neely, S. Pekarek and D. Stutts: IEEE Power Electron. Lett. 1 (2003) 69.

11 M. Bingabr, B. Espinoza-Varas and P. C. Loizou: Hearing Res. 241 (2008) 73.

12 P. A. Jespers: The $g_{\mathrm{m}} / I_{\mathrm{D}}$ Methodology, A Sizing Tool for Low-Voltage Analog CMOS Circuits (Springer, New York, 2010) Chap. 8.

13 R. R. Harrison and C. Charles: IEEE J. Solid-State Cir. 38 (2003) 958.

14 V. Chaturvedi and B. Amrutur: IEEE J. Emerg. Sel. Topic Cir. Syst. 1 (2011) 536.

15 S. Hur, Y. Jung, Y. H. Lee, W. J. Song and W. Kim: J. Korean Soc. Nondestruct. Test. 31 (2011) 522 . 